\title{
Meningitis and bacteraemia due to Listeria monocytogenes in compromised hosts
}

\author{
Mosche C. MaAyan \\ B.Sc., M.Sc. \\ Cilla Wajsman, \\ B.Sc., M.Sc. \\ *Yeshayahu Nitzan (ZaidenZaig) \\ B.Sc., M.Sc., Ph.D. \\ Medical Laboratories, Meir Hospital, Kfar-Saba, and \\ *Department of Life Sciences, Bar-Ilan University, Ramat-Gan, Israel
}

\begin{abstract}
Summary
The clinical history of 3 adult patients infected by Listeria monocytogenes is presented. One patient with chronic lymphatic leukaemia developed purulent meningitis; the 2 others had chronic renal failure and were undergoing routine haemodialysis. Of the latter, one developed meningitis and the other bacteraemia after receiving 2 blood transfusions. Immunosuppression, or the underlying disease of the hosts, probably played a role in permitting the infection to establish itself. The rural environment may also have been conducive to the transfer of this particular, rarely infectious, micro-organism to these patients.
\end{abstract}

\section{Introduction}

Infections due to Listeria monocytogenes are relatively rare and are usually limited to infants (about $80 \%$ of the cases) causing meningitis or meningoencephalitis (Gray and Killinger, 1966). Recently, however, Listeria infections were recorded in patients with predisposing disorders, such as cirrhosis, autoimmune diseases, renal transplants and malignancies (Bothone and Sierra, 1977). Since the micro-organism can be isolated from the healthy population of rural areas, it was presumed that asymptomatic human carriers play a role in transmitting it (Buchner and Schneierson, 1968). Very recently (1979), the authors isolated L. monocytogenes from 3 patients suffering from severe underlying diseases. To their knowledge, the present report records the first cases in which this micro-organism is involved in comprised hosts in the rural area in Israel.

\section{Microbiological methods}

Cerebrospinal fluid from a lumbar puncture was centrifuged and the sediment cultured on human blood agar plates. Blood samples were inoculated into trypticase soy broth and subcultured on blood agar plates. The plates were incubated aerobically under $10 \% \mathrm{CO}_{2}$ at $37^{\circ} \mathrm{C}$. Direct Gram staining was done on samples from the CSF sediment and colonies grown on blood agar. An established identification procedure was used (Lennette, Spaulding and Truant, 1974) which included the following tests: motility on semi-solid agar, determination of the fermentation pattern and a rapid slide test for presumptive identification of the $O$ antigen. An antibiogram determination was done on Wellcotest agar using discs of penicillin, ampicillin, methicillin, cephalothin, chloramphenicol, erythromycin and tetracycline.

\section{Case 1}

A 47-year-old man suffering from chronic renal failure and being treated by the haemodialysis unit since 1974, was admitted to the Meir Hospital with suspected meningitis. Physical examination on admission revealed stupor, apathy, aphasia, high sensitivity of the neck muscles and even slight stiffness of the neck. The rectal temperature was $38^{\circ} \mathrm{C}$. Laboratory examinations showed leucocytosis of WBC $12 \times 10^{9} / 1$. The results of lumbar puncture were WBC $0.72 \times 10^{9} / 1$, of which $95 \%$ were polymorphonuclear cells. In a direct smear of the CSF, Grampositive rods were seen. On blood agar plates, small haemolytic colonies grew and were stained as Grampositive rods. The micro-organism was identified as $L$. monocytogenes according to methods described below. Serological typing of the Listeria was not done in this case. The patient received $4 \times 10^{6} \mathrm{u}$. penicillin $G$ i.v., thrice/day for 3 days. He died in status epilepticus 4 days after admission.

\section{Case 2}

A 54-year-old woman suffering from chronic renal 
failure since 1972 and undergoing treatment in the haemodialysis unit was admitted to the hospital. Three days before admission she had received 2 blood transfusions and immediately thereafter had developed fever up to $38.5^{\circ} \mathrm{C}$, chills and leucocytosis. The patient's case history revealed that she had undergone (1973-1978) vagotomy, splenectomy, parathyroidectomy and partial gastrectomy. On admission, the patient suffered from severe pains in the shoulders extending into her hands. Physical examination indicated general weakness and chills. Neither stiffness of the neck nor other neurological sign was observed. The rectal temperature was $38-38.5^{\circ} \mathrm{C}$. Laboratory examinations showed a leucocytosis of WBC $11.7 \times 10^{9} / 1$, of which $81 \%$ were polymorphonuclear cells. In blood cultures L. monocytogenes type 4B was detected in 4 different cultures. The bacteraemic event was also associated with obstructive jaundice. The patient was treated with injections of cephazolin, $2 \mathrm{~g} /$ day. After initiation of the treatment, the temperature dropped and the chills ceased; the general condition improved. The patient was able to have haemodialysis every 2 days without any apparent difficulties. She was discharged from hospital after 15 days.

\section{Case 3}

A 58-year-old woman who had suffered from chronic lymphatic leukaemia for the last 3 years was admitted to hospital with fever and a history of headaches. Together with the chronic lymphatic leukaemia she also had hypogammaglobulinaemia and was being treated with prednisone and chlorambucyl. As a result of this therapy her bone marrow was depressed - the number of segmented cells had declined to $1 \%$ and the number of lymphocytes was $91 \%$. Her haemoglobin was $10.2 \mathrm{~g} / \mathrm{dl}$, the haematocrit $31 \%$ and the leucocyte count WBC $35 \times 10^{2} / 1$. Several hours after admission, meningeal signs began to appear: enlarged axillary glands, stiffness of the neck, Brudzinski's and Kernig's signs. The rectal temperature was $37 \cdot 7^{\circ} \mathrm{C}$. The lumbar puncture was cloudy and contained WBC $1.768 \times 10^{9} / 1,56 \%$ of which were $\mathrm{T}$ lymphocytes; the protein content was $4.55 \mathrm{~g} / \mathrm{l}$. In a culture of the fluid the presence of $L$. monocytogenes was detected, and these were further identified as being of type 1B. The patient was treated with i.v. erythromycin $3 \mathrm{~g} /$ day for 29 days. After 2 days of this medication, the fever subsided, the headaches disappeared and her general condition improved. Erythromycin was used because the patient was hypersensitive to penicillins. She was discharged from the hospital after 30 days.

\section{Discussion}

L. monocytogenes has sometimes been called the 'Cinderella' among the pathogenic bacteria (Gray and Killinger, 1966; Bothone and Sierra, 1977). This is probably due to its opportunistic habits of invasion. In the past, Listeria was thought to be the cause of repeated abortions and perinatal infections because it had been isolated from the female genital tract (Rappaport et al., 1960). Later reports documented Listeria infections in patients with underlying diseases, particularly those affecting the immune system (Louria et al., 1967; Simpson, Leddy and Hare, 1967). In these patients, L. monocytogenes may cause meningitis, conjunctivitis, septicaemia endocarditis or osteomyelitis (Gray and Killinger, 1966). The predisposing features to L. monocytogenes infection which were encountered in the present patients were: chronic lymphatic leukaemia and chronic renal failure; the age range of the patients was 47-58 years, and they had suffered 3-7 years from the underlying disease. The authors have no positive information about the source of infection or the manner of entry. In one case it may have been through a blood transfusion; in the other 2, one may assume that asymptomatic carriers played a role in transmitting the disease. Patients from rural areas surrounding this hospital who, in addition, are exposed for a prolonged period of time to procedureso such as haemodialysis, operations, transfusions, etc may be more susceptible to transmission from the environment. Listeria is more widespread than generally assumed, but the authors are usually unaware of it since the general healthy population is not affected, but the targets are mainly the high risk patients (Larsson, 1978). From the cases reported it is difficult to state clearly whether the infection is nosocomial or has been transmitted directly from the rural environment. The facts may show that both ways have actually happened, but the underlying disease is the important factor, since it permits the infection to occur.

There is still a difficulty in differentiating colonies of $L$. monocytogenes from saprophytic diphtheroids or other corynebacteria on the usual culture plates. This problem is still a major obstacle in detecting $L$. monocytogenes and to understand its epidemiology. The absence of an efficient, selective method for isolating $L$. monocytogenes seriously impedes the study of listeriosis (Kramer and Jones, 1969). In the present study diagnosis was established by cultures and by demonstrating the typical colonies or the morphology of the micro-organisms. The appearance of colonies with a blue-green hue on colourless solid media or umbrella-shaped colonies on semi-solid agar in $25^{\circ} \mathrm{C}$ is very reliable for early identification of this organism. The full battery of recommended biochemical tests are more than adequate for presumptive identification (Lennette et al., 1974). Final identification was established by serotyping the isolates. 
Types 1B and 4B, which were found in these patients, are believed to be the serotypes most frequently causing infections (Bothone and Sierra, 1977). Concerning antibiotic therapy, penicillin, ampicillin, tetracycline or erythromycin were often used as treatment of choice (Wiggins, Albritton and Feeley, 1978). For the present patients, erythromycin and cephazolin proved efficient. The Listeria isolates were all sensitive to penicillin, ampicillin, cephalothin, tetracycline and chloramphenicol. Only one strain was resistant to methicillin.

Because of the difficulties in identifying $L$. monocytogenes and the probability of misidentifying the cause of infection from clinical specimens, the diagnosis of Listeria infections demands alertness. Since the incidence of attack by an opportunist such as Listeria is always greater in high risk patients in rural areas, a higher degree of awareness is required in these cases.

\section{Acknowledgment}

We are grateful to Professor L.A. Rozenszajn for his advice and wish to thank $H$. Niv and H. Arielli for skilful technical assistance.

\section{References}

Bothone, E.J. \& SIERRA, M.F. (1977) Listeria monocytogenes: Another look at the 'Cinderella among pathogenic bacteria'. Mount Sinai Journal of Medicine, 44, 42.
Buchner, L.H. \& Schneierson, S.S. (1968) Clinical laboratory aspects of Listeria monocytogenes infections. American Journal of Medicine, 45, 904.

Gray, M.L. \& KILlinger, A.H. (1966) Listeria monocytogenes and listeric infections. Bacteriological Review, 30, 309.

Kramer, P. \& Jones, D. (1969) Media selective for Listeria monocytogenes, Journal of Applied Bacteriology, 32, 381.

LARsson, S. (1978) Clinical aspects on 64 cases of juvenile and adult listeriosis in Sweden. Acta medica scandinavica, 204, 503.

Lennette, E.H., Spaulding, E.H. \& Truant, J.P. (1974) In: Manual of Clinical Microbiology 2nd edn, pp. 136-138. American Society for Microbiology, Washington D.C.

Louria, D.B., Hensle, T., Armstrong, D., Collins, H.S., Blevins, A., Krugman, A. \& Buse, M. (1967) Listeriosis complicating malignant diseases. A new association. Annals of Internal Medicine, 67, 261.

RAPPAPORT, F., RABINOWITZ, M., ToAfF, R. \& KROChIK, N. (1960) Genital listeriosis as a cause of repeated abortion. Lancet, i, 1273.

Simpson, J.F., LedDy, J.P. \& HARE, J.D. (1967) Listeriosis complicating lymphoma. Report of four cases and interpretative review of pathogenic factors. American Journal of Medicine, 43, 39.

Wiggins, G.L., Albritton, W.L. \& Feeley, J.C. (1978) Antibiotic susceptibility of clinical isolates of Listeria monocytogenes. Antimicrobial Agents and Chemotherapy, 13, 854. 